Supporting Information

\title{
Nonthermal Plasma-Synthesized Phosphorus-Boron co-Doped Si Nanocrystals: A New Approach to Non-Toxic NIR-Emitters
}

\author{
Rens Limpens*\#, Gregory F. Pach", and Nathan R. Neale* \\ National Renewable Energy Laboratory, Golden, Colorado 80401, United States \\ (*Corresponding author, ${ }^{*}$ Equal contribution)
}

\begin{tabular}{|c|c|c|c|c|c|c|c|c|c|}
\hline NC size & Tube Size (ID) & $30 \% \mathrm{~B}_{2} \mathrm{H}_{6} / \mathrm{H}_{2}$ & $\mathrm{PH}_{3}$ & $100 \% \mathrm{SiH}_{4}$ & $10 \% \mathrm{SiH}_{4} / \mathrm{He}$ & $\mathrm{Ar}$ & $\mathrm{H}_{2}$ & Pressure & Power \\
\hline $2.9 \mathrm{~nm}$ & $19 \mathrm{~mm}$ & $0.7 \mathrm{sccm}$ & $1.4 \mathrm{sccm}$ & $8.6 \mathrm{sccm}$ & N/A & $48 \mathrm{sccm}$ & $168 \mathrm{sccm}$ & 1.0 Torr & $250 \mathrm{~W}$ \\
\hline $3.0 \mathrm{~nm}$ & $19 \mathrm{~mm}$ & $2.4 \mathrm{sccm}$ & $1.4 \mathrm{sccm}$ & $8.6 \mathrm{sccm}$ & N/A & $48 \mathrm{sccm}$ & $168 \mathrm{sccm}$ & 1.5 Torr & $250 \mathrm{~W}$ \\
\hline $3.2 \mathrm{~nm}$ & $19 \mathrm{~mm}$ & $1.2 \mathrm{sccm}$ & $1.4 \mathrm{sccm}$ & $8.6 \mathrm{sccm}$ & N/A & $48 \mathrm{sccm}$ & $168 \mathrm{sccm}$ & 1.5 Torr & $250 \mathrm{~W}$ \\
\hline $3.4 \mathrm{~nm}$ & $19 \mathrm{~mm}$ & $0.7 \mathrm{sccm}$ & $1.4 \mathrm{sccm}$ & $8.6 \mathrm{sccm}$ & N/A & $48 \mathrm{sccm}$ & $168 \mathrm{sccm}$ & 1.5 Torr & $250 \mathrm{~W}$ \\
\hline $4.0 \mathrm{~nm}$ & $19 \mathrm{~mm}$ & $0.7 \mathrm{sccm}$ & $1.4 \mathrm{sccm}$ & $8.6 \mathrm{sccm}$ & $\mathrm{N} / \mathrm{A}$ & $48 \mathrm{sccm}$ & $168 \mathrm{sccm}$ & 2.0 Torr & $250 \mathrm{~W}$ \\
\hline $4.7 \mathrm{~nm}$ & $19 \mathrm{~mm}$ & $0.7 \mathrm{sccm}$ & $1.4 \mathrm{sccm}$ & $8.6 \mathrm{sccm}$ & $\mathrm{N} / \mathrm{A}$ & $48 \mathrm{sccm}$ & $168 \mathrm{sccm}$ & 2.5 Torr & $250 \mathrm{~W}$ \\
\hline $5.0 \mathrm{~nm}$ & $19 \mathrm{~mm}$ & $0.7 \mathrm{sccm}$ & $1.4 \mathrm{sccm}$ & $8.6 \mathrm{sccm}$ & N/A & $48 \mathrm{sccm}$ & $168 \mathrm{sccm}$ & 3.0 Torr & $250 \mathrm{~W}$ \\
\hline $5.0 \mathrm{~nm}$ & $19 \mathrm{~mm}$ & $1.2 \mathrm{sccm}$ & $1.4 \mathrm{sccm}$ & $8.6 \mathrm{sccm}$ & N/A & $48 \mathrm{sccm}$ & $168 \mathrm{sccm}$ & 1.0 Torr & $250 \mathrm{~W}$ \\
\hline $5.3 \mathrm{~nm}$ & $19 \mathrm{~mm}$ & $0.7 \mathrm{sccm}$ & $1.4 \mathrm{sccm}$ & $8.6 \mathrm{sccm}$ & $\mathrm{N} / \mathrm{A}$ & $48 \mathrm{sccm}$ & $168 \mathrm{sccm}$ & 3.5 Torr & $250 \mathrm{~W}$ \\
\hline $7.1 \mathrm{~nm}$ & $6.3 \mathrm{~mm}$ to $19 \mathrm{~mm}$ & $1.2 \mathrm{sccm} *$ & $0.7 \mathrm{sccm}$ & N/A & $30 \mathrm{sccm}$ & $30 \mathrm{sccm}$ & $3 \mathrm{sccm}$ & 3.0 Torr & $100 \mathrm{~W}$ \\
\hline $7.3 \mathrm{~nm}$ & $6.3 \mathrm{~mm}$ to $19 \mathrm{~mm}$ & $2.4 \mathrm{sccm}^{*}$ & $0.7 \mathrm{sccm}$ & $\mathrm{N} / \mathrm{A}$ & $30 \mathrm{sccm}$ & $30 \mathrm{sccm}$ & $3 \mathrm{sccm}$ & 3.0 Torr & $100 \mathrm{~W}$ \\
\hline & & & & & & & & & \\
\hline
\end{tabular}

Table S1. Synthetic methods for all PB:Si-H NCs shown in this report 


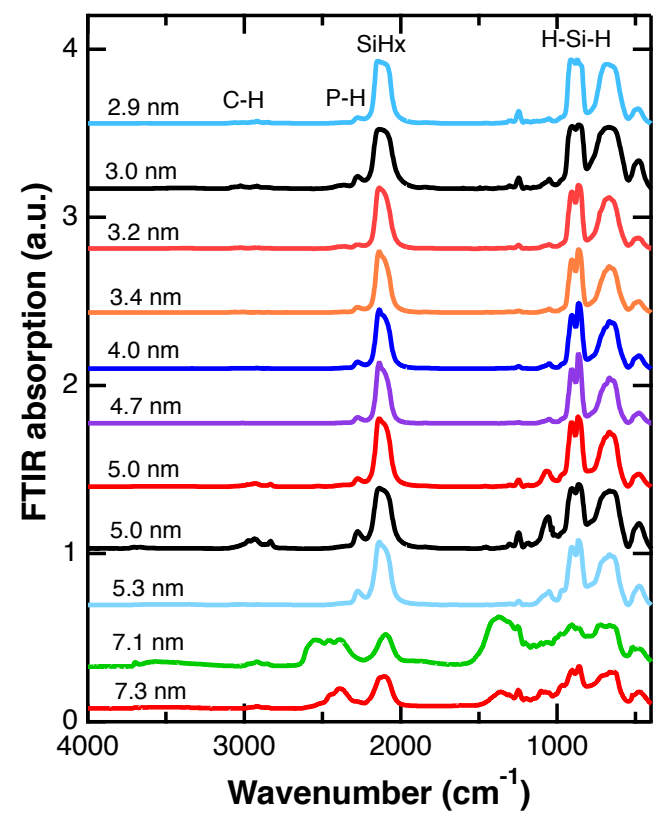

Figure S1. FTIR spectra of all PB:Si-H with diameters ranging from $D_{N C}=2.9-7.3 \mathrm{~nm}$.

a)

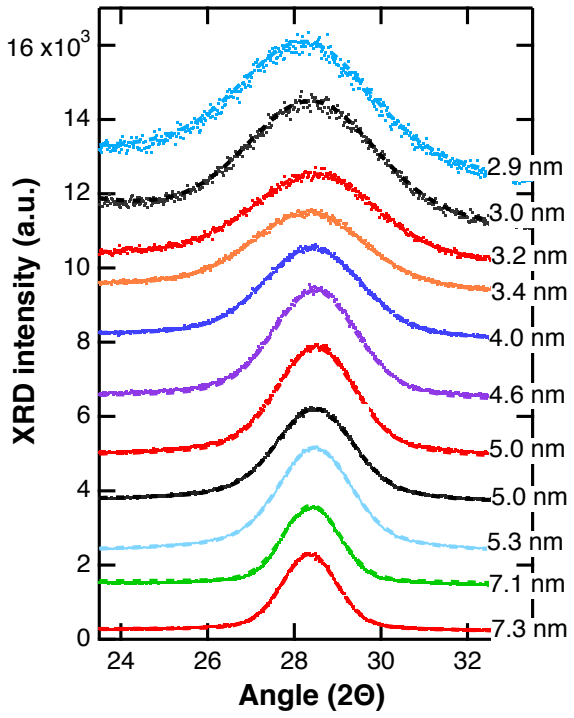

b)

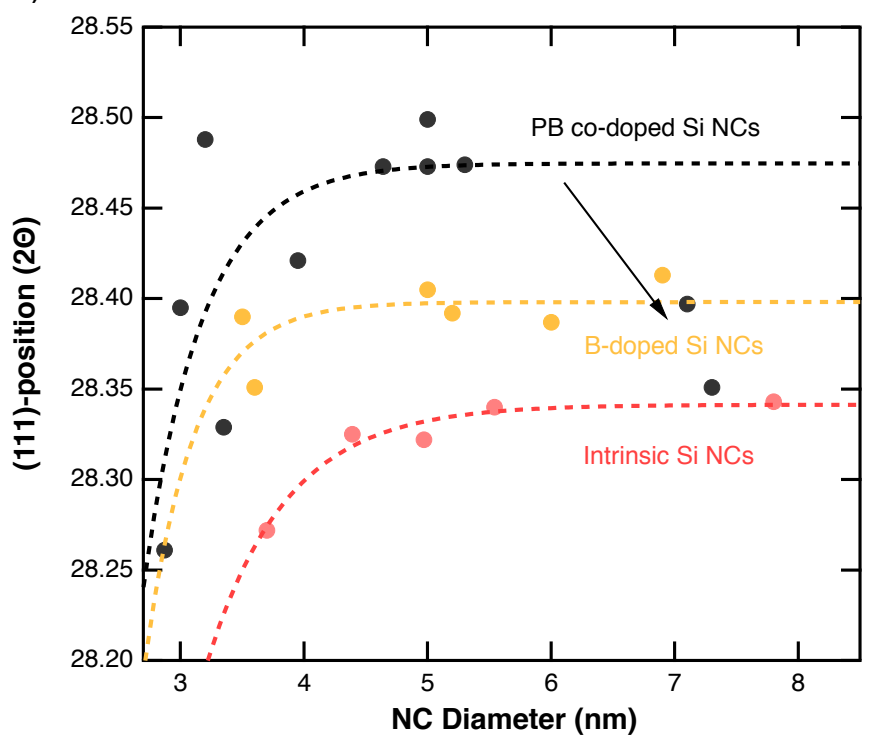

Figure S2. a) XRD patterns of the crystalline Si (111)-diffraction peak for all PB:Si-H NC samples in this study $\left(D_{N C}=2.9-7.3 \mathrm{~nm}\right)$. b) (111) XRD peak position versus $N C$ diameter for plasmasynthesized intrinsic and B-doped NC systems of previous work, ${ }^{4}$ and for PB:Si-H NCs in this work. The arrow is a visual aid for the positions of the two largest PB:Si-H NC samples.

All PB:Si NCs show increased (111)-peak positions in direct comparison to similarly sized Bdoped or intrinsic Si NC systems. Higher diffraction angles indicate smaller lattice constant and this observation therefore aligns with substitutional incorporation of the B-atoms, which have a significantly smaller atomic radius $(85 \mathrm{pm})$ than that of $\mathrm{P}(100 \mathrm{pm})$ and $\mathrm{Si}(110 \mathrm{pm})$ atoms. It is worth noting that although the $\mathrm{P}$ atom is slightly smaller than $\mathrm{Si}$, heavy substitutional P-doping of 
Si NCs does not cause a measurable shift in the lattice constant ${ }^{4}$, the shift measured in this work is therefore solely ascribed to the substitutional incorporation of B atoms in the NC lattice.

We determine the interplanar spacing $d$ by:

$$
\lambda=2 d_{h k l} \sin \theta_{h k l}
$$

Subsequently, the lattice constant $a$ is determined by assuming a cubic packing structure (FCC in this case) in which $\mathrm{a} 1=\mathrm{a} 2=\mathrm{a} 3$ (cube sites of unit cell):

$$
\frac{1}{d^{2}}=\frac{h^{2}+k^{2}+l^{2}}{a^{2}}
$$

Taking the (111)-diffraction peak $(\mathrm{h}=1, \mathrm{k}=1,1=1)$ and calculating $d$ from its $\theta$ position, we arrive at the lattice constant $a$, which is 5.431, 5.455 and $5.457 \AA$ for PB:Si, B:Si and intrinsic $\mathrm{Si}$ NCs in the large diameter limit $\left(D_{\mathrm{NC}}>6 \mathrm{~nm}\right)$, respectively. Note the tendency to smaller angles (bigger lattice constants) for smaller NC sizes, for all NC systems. This most likely results from an increased surface energy for smaller NCs that deforms the crystalline Si lattice. Interestingly, the fact that the PB co-doped Si NCs have smaller lattice constants than heavily B-doped systems indicate that substitutional B incorporation is more effective in the presence of the co-dopant $\mathrm{P}$, perhaps due to decreases in electrostatic repulsion during the nucleation and agglomeration growth process.

Estimated Diameters from 249 PB:Si NCs

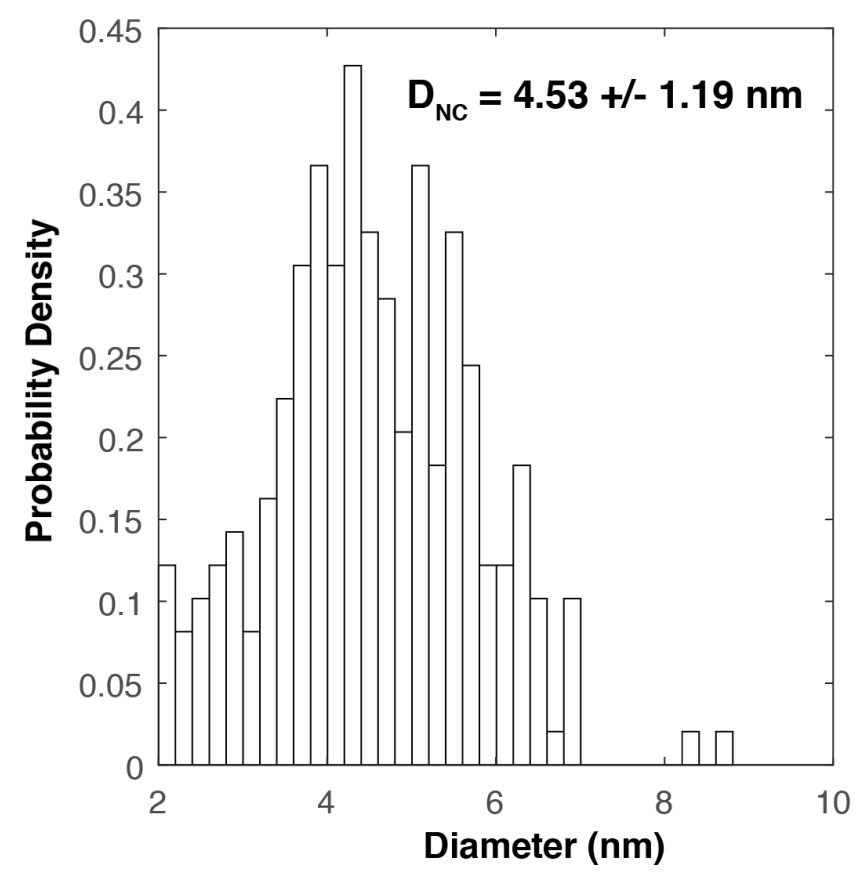

Figure S3. Sizing histogram from TEM analysis of PB:Si-H NCs. Scherrer analysis of this sample estimated a size of $D_{N C}=4.6 \mathrm{~nm}$. 


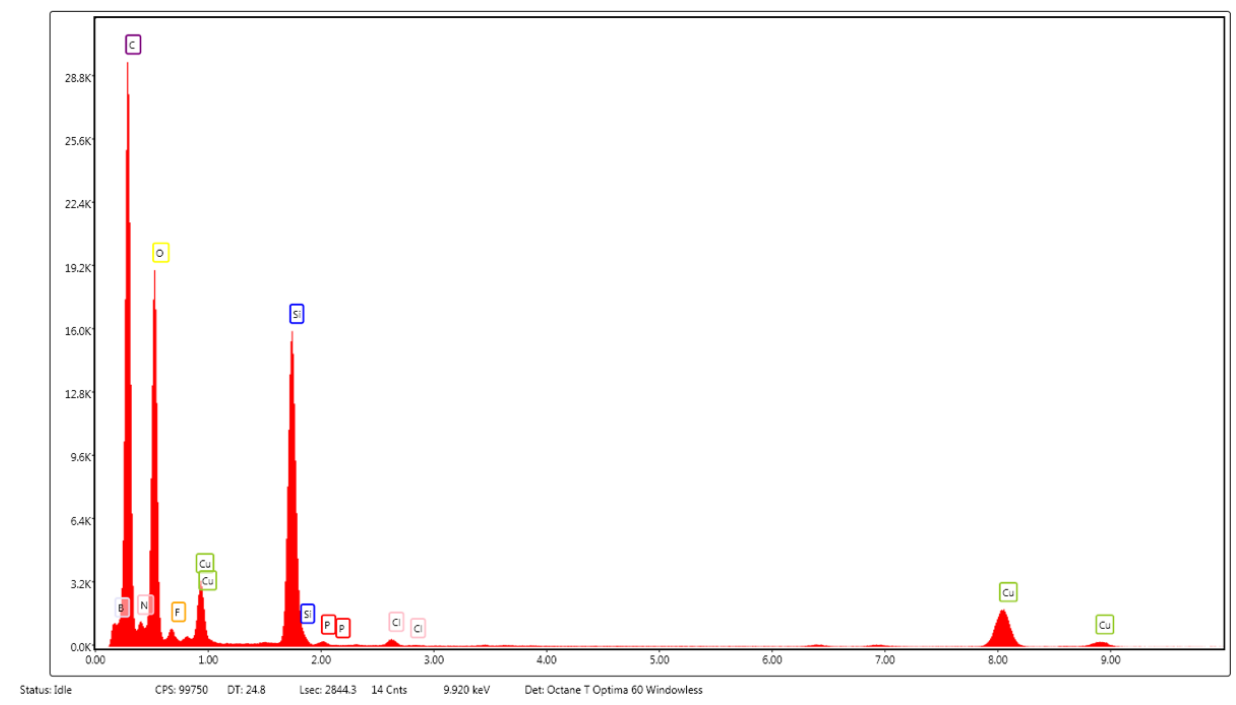

Figure S4. EDS spectrum from a PB:Si-R sample. Phosphorus and boron peaks are both present.

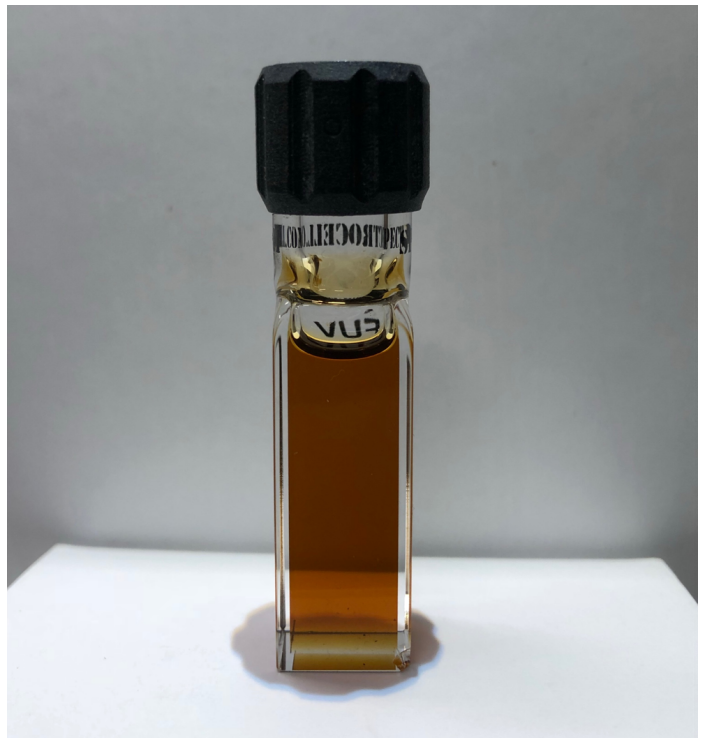

Figure S5. Spontaneous solution formation of PB:Si-H in N-methylpyrrolidone (a strong electron donor solvent). 


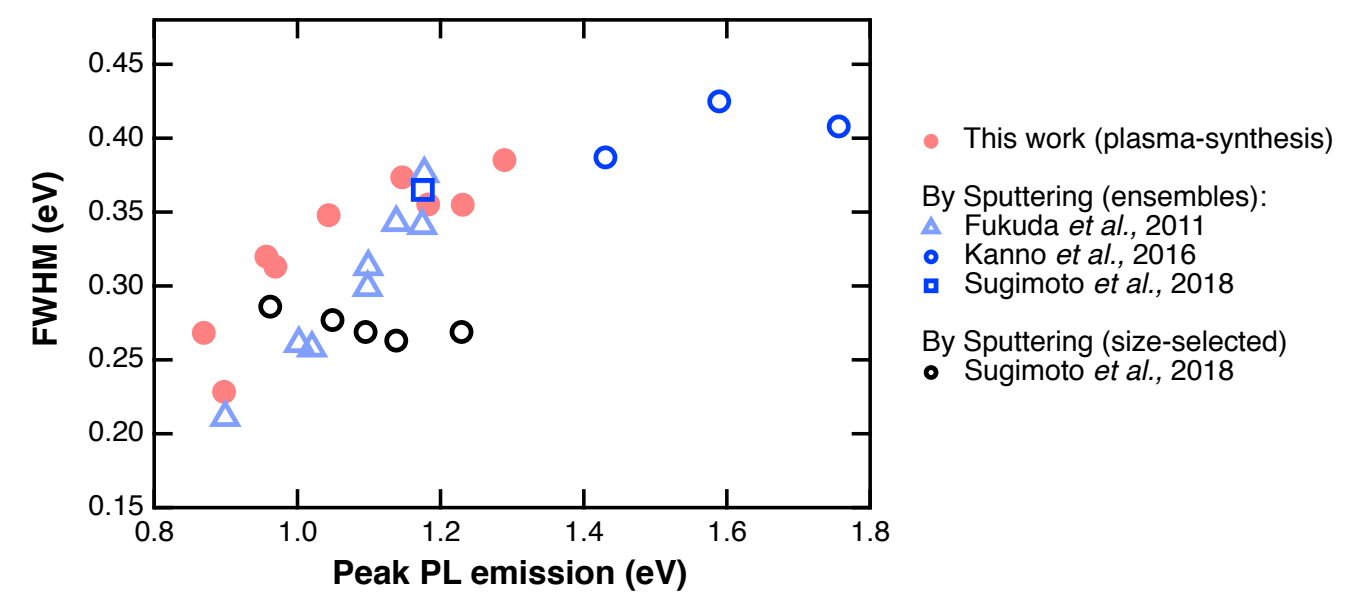

Figure S6. FWHM evaluation of PB:Si-H as a function of PL peak emission energy (red dots). Blue symbols show thermodynamically grown PB:Si NCs (blue triangles, ${ }^{5}$ blue open circles ${ }^{6}$, blue squares $^{7}$ and size-selected ensembles in the black circles $\left.^{7}\right)$.

Evaluation of the FWHM (closed red circles, Figure S6) of the plasma-synthesized PB:Si-H show similar values compared to thermodynamically grown systems (blue symbols). Upon sizeselection, these distributions could be slightly narrowed for the thermodynamically grown systems (black open circles). ${ }^{7}$ The antisolvent precipitation approach used in their work should be transferable to our system as well. 

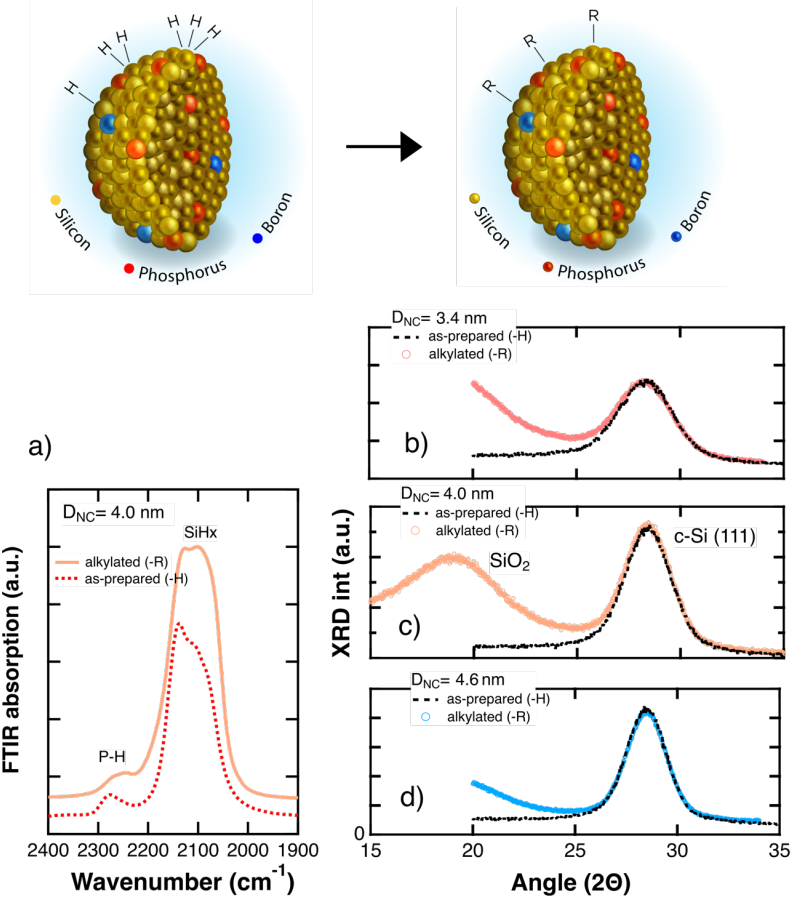

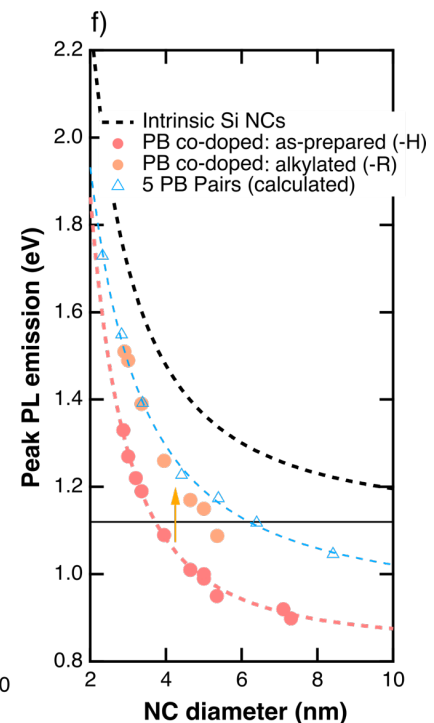

Figure S7. Characterization of alkylated PB:Si NCs, in the scheme depicted by the " $-R$ " surfacegroup. a) FTIR spectra, $b$-d) XRD patterns, e) PL spectra and f) peak PL emission as a function of the NC diameter. Artistic Scheme: Credit Alfred Hicks, NREL.

The PB co-doped Si NCs are functionalized with alkyl groups by reaction with 1-octadecene following thermal-induced radical initiation, details are provided in our previous publication. ${ }^{2}$ XRD-pattern comparison between as-prepared and alkylated NC systems (panel b-d) show similar broadening of the (111) diffraction peak, indicating that no size selective processes take place during the functionalization process. The functionalized NCs show slightly blue-shifted PL spectra (panel e and f) compared to the as-prepared NC ensembles, as expected for the increased electron density provided by the addition of alkyl ligands. The XRD patterns show that the observed PL shift cannot be solely attributed to size-selective precipitation during post-functionalization solvent/anti-solvent washing. The origin of this shift likely stems from multiple sources. NCs with a larger concentrations of surface dopants will remain in solution during the washing procedure as acetonitrile (the anti-solvent) will coordinate to surface boron sites on the NCs. As a result, the less heavily doped systems increase their dominance in these ensemble measurements, leading to a blue-shift of the emission. The blue shifted emission can also reflect an increase in confinement within functionalized NCs. Our previous work has shown a compressed wavefunction as a result of alkyl surface functionalization ${ }^{2}$ and other reports have shown similar results. ${ }^{8,9}$ 
Scheme. Fiber-coupled integrating sphere

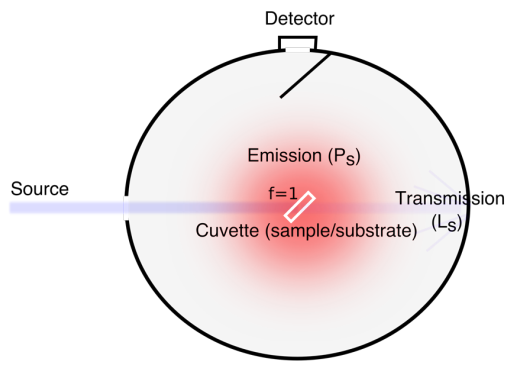

b)

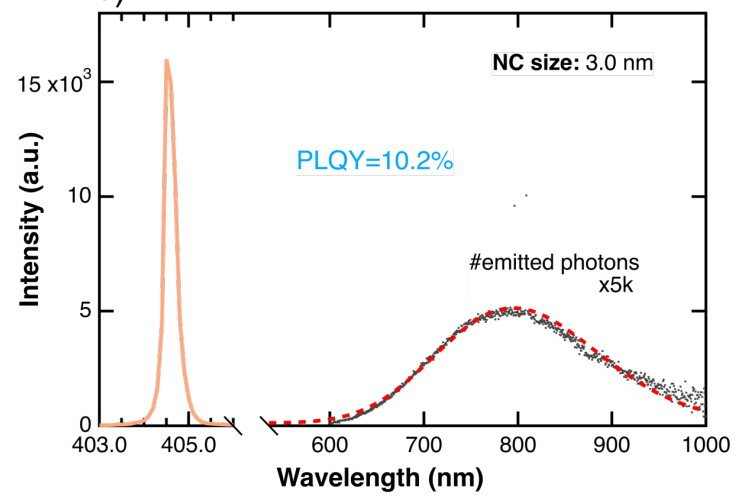

d)

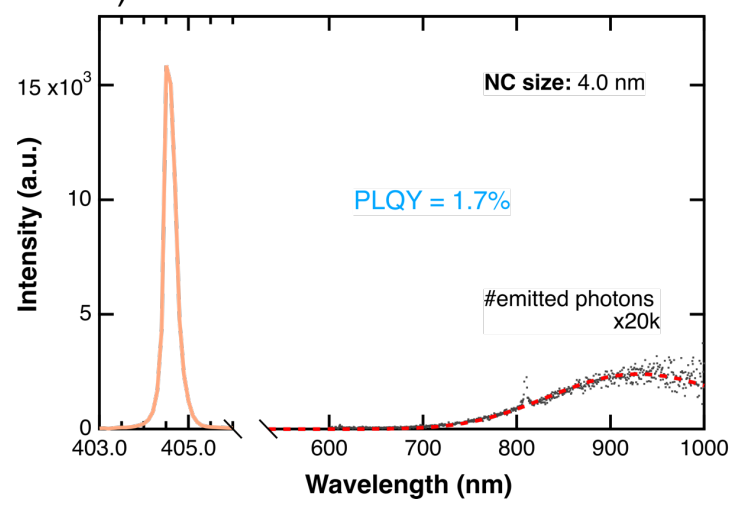

a)

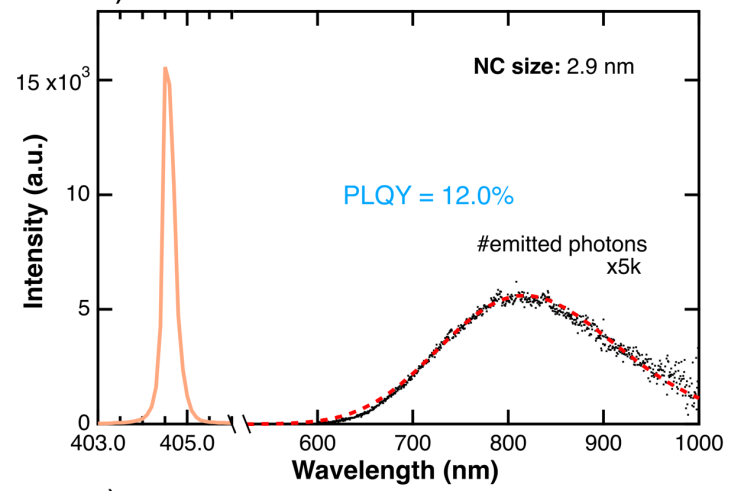

c)

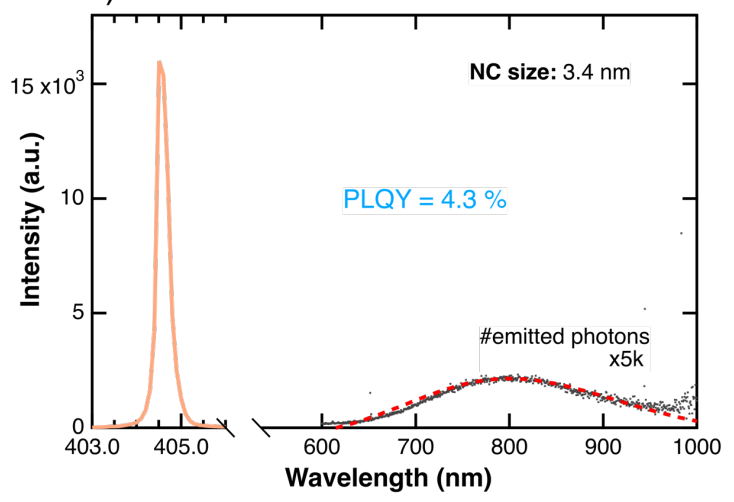

e)

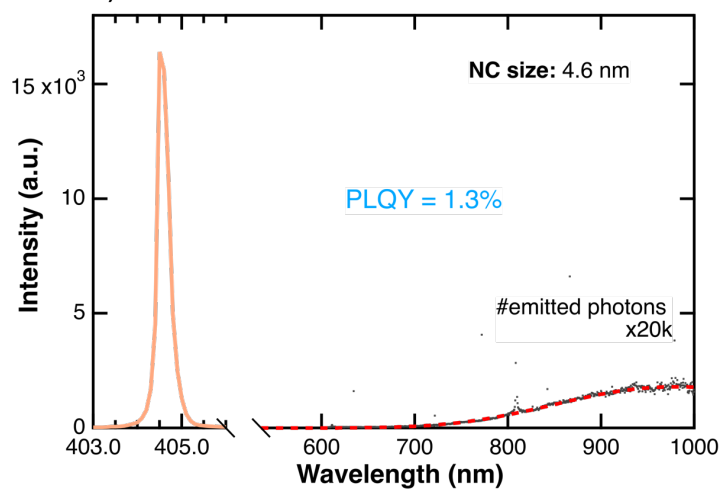

Figure S8. Absolute PLQY measurements for PB:Si-R by the method described in Ref[10]. (as illustrated by the scheme). a-e) Absorption (orange line) and emission (black dots) spectra of the $P L Q Y$ measurements for $D_{N C}=2.9-4.6 \mathrm{~nm}$. The emission can be approximated by a lognormal fitting function (red dashed line). The scheme of the experimental setup is taken from Ref. ${ }^{11}$

Absolute PLQY measurements are performed following the technique described by Mangolini et al., ${ }^{10}$ with:

$$
\frac{P_{s}-P_{b}}{-\left(L_{s}-L_{S}\right)}=P L Q Y,
$$


with $P$ and $L$ the spectrally integrated photon counts of the photoluminescence and excitation light, respectively. Subscripts $s$ and $b$ refer to the sample and substrate (i.e., baseline) measurements. $f$ in the scheme refers to the fraction of light that directly hits the sample (unity in our experimental setup). The PLQY measurements are thus performed by conducting a measurement on both the sample and a reference (cuvette with the solvent only, TCE in this case). Subtraction of the measurements quantifies the number of absorbed photons in the excitation window (403-406 nm, in orange) and the number of emitted photons in the PL window (550-1000 nm, in black). Optical density (OD) of all samples was around 1 , at $405 \mathrm{~nm}$ excitation $\left(O D=-\log _{10}[T]\right)$. Various cuvette thicknesses were used (with excitation depths of $1 \mathrm{~cm}$ and $2 \mathrm{~mm}$ ), no differences in PLQY values are observed between these two variations.

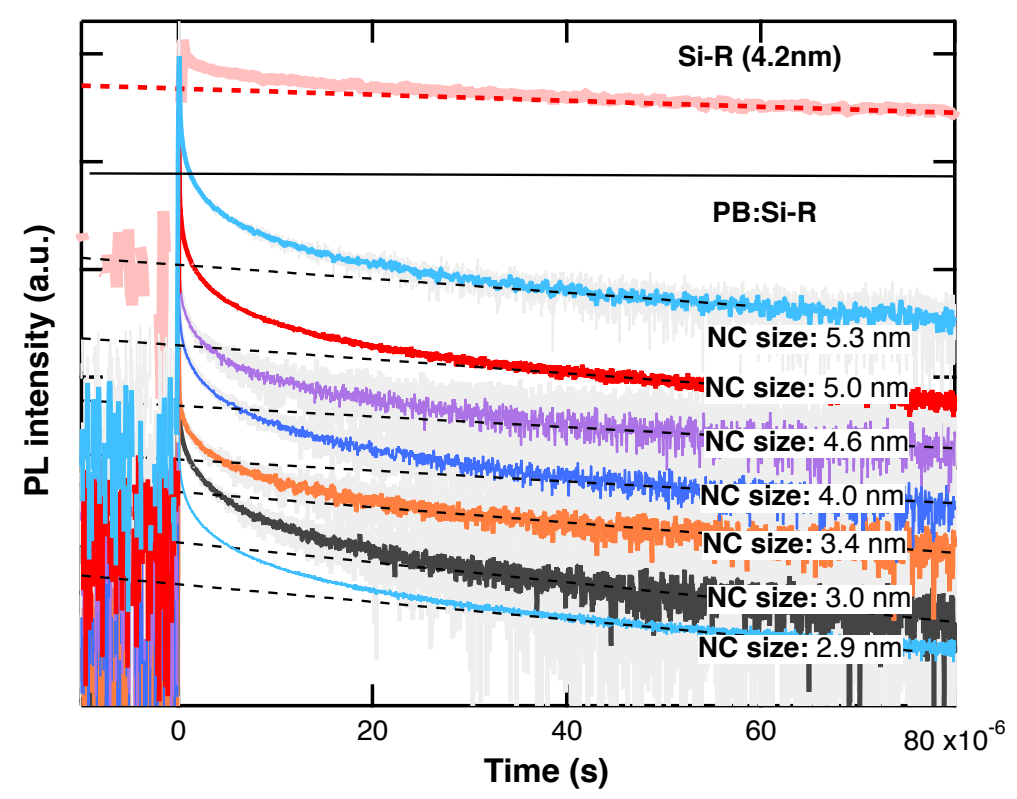

Figure S9. Time-resolved PL (TRPL) traces for PB:Si-R with $D_{N C}=2.9-7.1 \mathrm{~nm}$, a log-lin scale is used. An excitation wavelength of $\lambda_{\text {pump }}=532 \mathrm{~nm}$ is used. The black dashed lines are singleexponential fits. For reference, we incorporated a typical TRPL trace of an intrinsic Si-R NC ensemble $\left(D_{N C}=4.2 \mathrm{~nm}\right)$, taken from Ref. ${ }^{2}$ 
a)

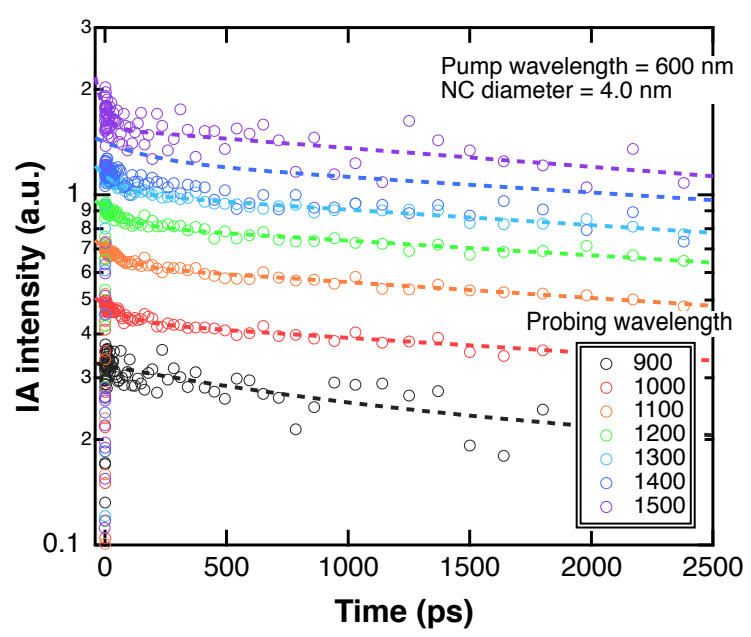

b)

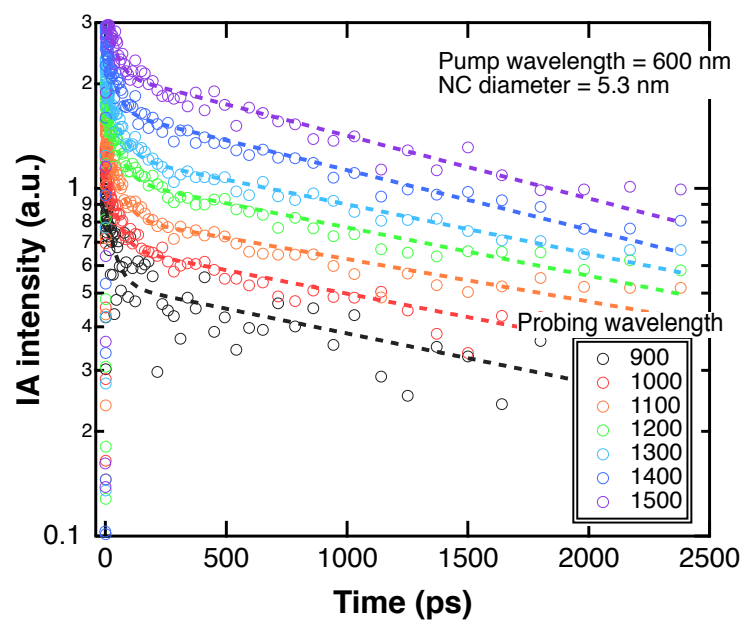

Figure S10. Ultrafast carrier dynamics by IA spectroscopy on PB:Si-R with $D_{N C}=4.0 \mathrm{~nm}(\mathrm{a})$ and $5.3 \mathrm{~nm}$ (b), with $\lambda_{\text {pump }}=600 \mathrm{~nm}$ and $\lambda_{\text {probe }}=900-1500 \mathrm{~nm}$. Dashed lines are double exponential fits as guides to the eye.

Figure S10 displays the probing wavelength dependence for two PB co-doped NC systems: below the critical NC size $(5.0 \mathrm{~nm}$, panel a) and above the critical size $(5.3 \mathrm{~nm}$, panel b). Both systems exhibit kinetics that are independent of the probing wavelength, indicating the absence of any state filling effects. This is in line with a previous study of us on heavily singly-doped Si NCs (both nand p-type), ${ }^{4}$ in which we could not observe any state-filling bleaching effects.

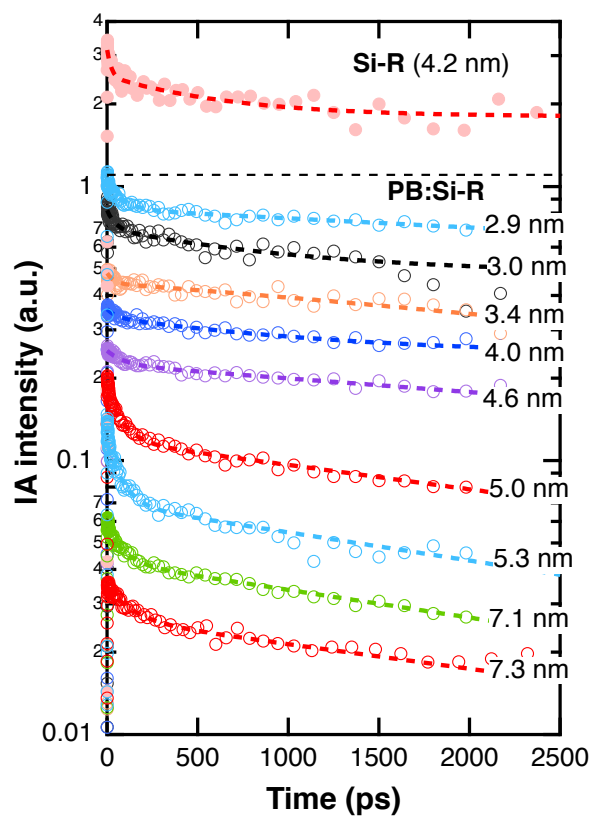

Figure S11. All TA traces (in the single-exciton excitation regime) for PB:Si-R NCs, with pump and probe wavelengths $\lambda_{\text {pump }}=600 \mathrm{~nm}$ and $\lambda_{\text {probe }}=1200 \mathrm{~nm}$, respectively. 
a)

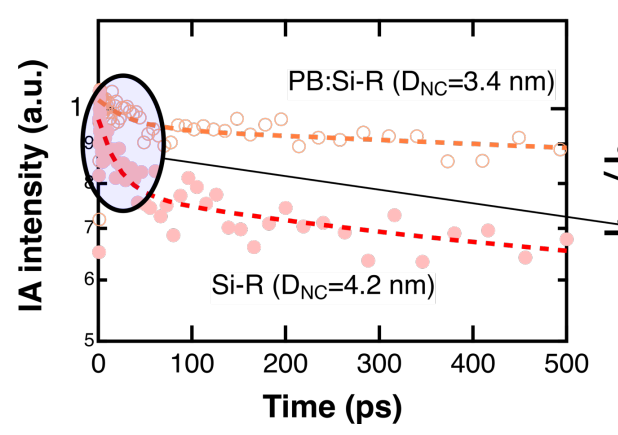

b)

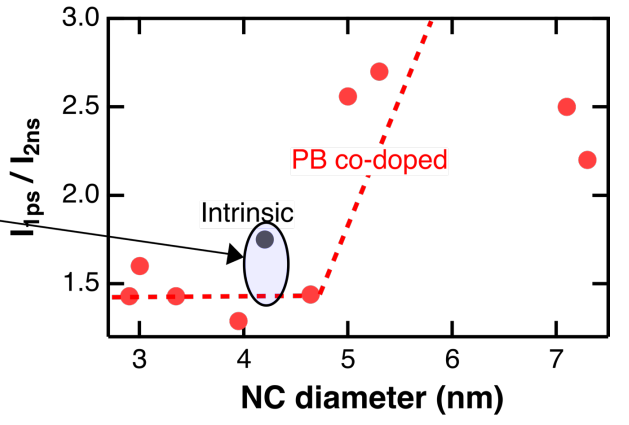

Figure S12. a) Ultrafast kinetics by pump-probe IA spectroscopy $\left(\lambda_{\text {pump }}=600 \mathrm{~nm}\right.$ and $\lambda_{\text {probe }}=$ $1200 \mathrm{~nm})$ of PB:Si-R $\left(D_{N C}=3.4 \mathrm{~nm}\right)$ and intrinsic Si-R NCs $\left(D_{N C}=4.2 \mathrm{~nm}\right)$. b) Ratio of the intensity at 1 ps and $2 \mathrm{~ns}\left(I_{1 p s} / I_{2 n s}\right)$, illustrating the strength of the nonradiative decay channels in the initial 2 ns after photoexcitation.

Figure S12a displays the ultrafast carrier dynamics within the first 500 ps ns of creation within PB:Si-R NCs $\left(\mathrm{D}_{\mathrm{NC}}=3.4 \mathrm{~nm}\right)$ in comparison to a typical trace of intrinsic $\mathrm{Si}-\mathrm{R}$ NCs $\left(\mathrm{D}_{\mathrm{NC}}=4.2\right.$ $\mathrm{nm}$ ). The kinetics are nearly identical, with a minor difference in the initial $\sim 30$ ps where the intrinsic Si NCs display a fast recombination pathway indicative of nonradiative surface recombination effects. Quantification of the total nonradiative quench over the initial $2 \mathrm{~ns}$ is shown in Figure S12b.

Note that the $\sim 30$ ps recombination channel also exists in the carrier dynamics of the PB:Si systems, but with much lower intensity/magnitude. To this end we note that, while glass-matrix grown Si NCs are known for their surface dangling bonds that can be passivated through $\mathrm{P}^{12}$ or $\mathrm{B}^{13}$ surface atoms, our previous work ${ }^{3}$ showed that these plasma-grown $\mathrm{Si}$ NCs have fully passivated surfaces. The reduced quench in the initial $30 \mathrm{ps}$ is therefore unlikely a result of a higher degree of surface passivation but might be originating from surface reconstruction/relaxation by $\mathrm{P}$ and B atoms that somehow enables better NC qualities. Our prior theoretical work, for example, shows that $\mathrm{B}$ and $\mathrm{P}$ exhibit lower energies at the surface relative to the core for hydrogen deficient surfaces ${ }^{14}$ that may be related to this result. Future studies are necessary to elucidate this effect. 

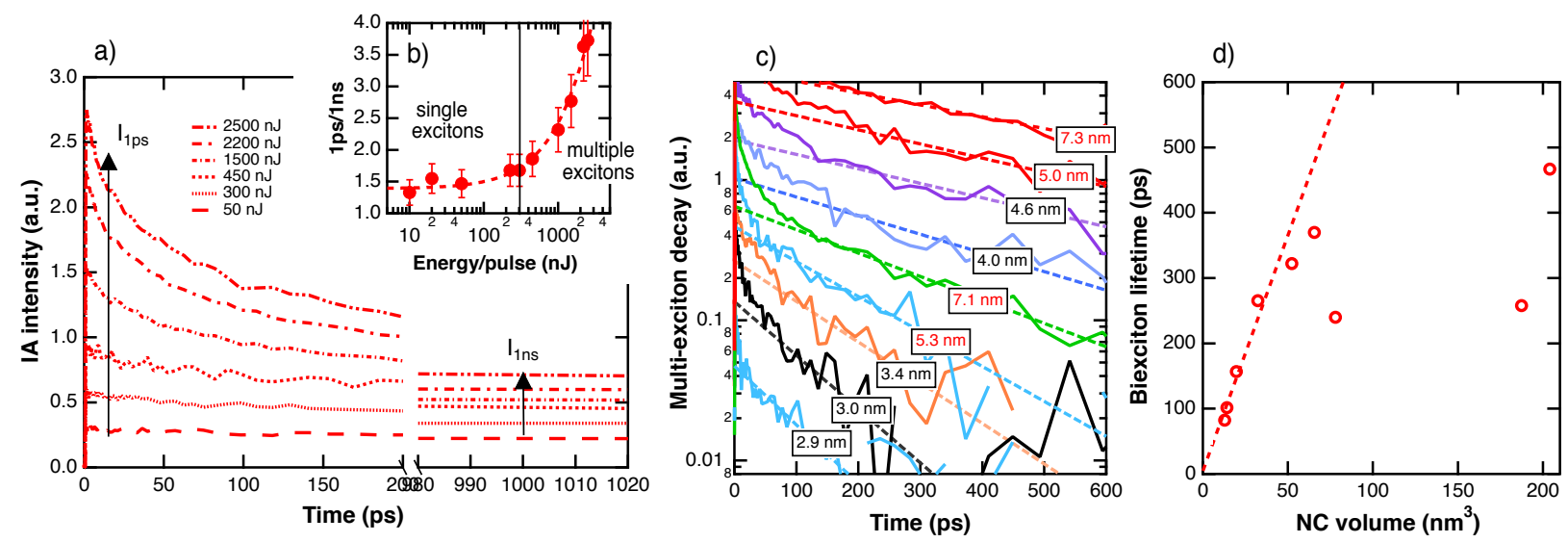

Figure S13. Derivation of the biexciton lifetimes following Ref. ${ }^{14}$. a) kinetics of PB:Si-R with $D_{N C}$ $=7.3 \mathrm{~nm}$, for different excitation powers. b) Quantification of the single-and multi-exciton excitation regime, by plotting the ratio intensity $\left(I_{1 p s} / I_{1 n s}\right)$ of the kinetics from panel (a) as a function of the energy per excitation pulse (in $n J)$. c) Multi-exciton decay kinetics for PB:Si-R with $D_{N C}=2.9-7.3 \mathrm{~nm}$, constructed by subtracting the kinetics in the single-exciton excitation regime from the multi-exciton excitation regime (illustrated in panel b), following Ref. ${ }^{15}$. The traces are ordered based on their biexciton lifetime. Dashed lines are single-exponential fits that reflect the biexciton lifetime. d) All fitted biexciton lifetimes as a function of the NC volume. The red dashed line is a linear fit and indicates the typical linear volume dependence of the biexciton lifetime.

\section{References}

(1) Wheeler, L. M.; Anderson, N. C.; Palomaki, P. K. B.; Blackburn, J. L.; Johnson, J. C.; Neale, N. R. Silyl Radical Abstraction in the Functionalization of Plasma-Synthesized Silicon Nanocrystals. Chem. Mater. 2015, 27, 6869-6878.

(2) Carroll, G. M.; Limpens, R.; Neale, N. R. Tuning Confinement in Colloidal Silicon Nanocrystals with Saturated Surface Ligands. Nano Lett. 2018, 18, 3118-3124.

(3) Limpens, R.; Neale, N. R. Free Electron-Driven Photophysics in n-Type Doped Silicon Nanocrystals. Nanoscale 2018, 10, 12068-12077.

(4) Limpens, R.; Pach, G. F.; Mulder, D.; Neale, N. R. Size-Dependent Asymmetric Auger Interactions in Plasma-Produced n- and p-Type Doped Silicon Nanocrystals. J. Phys. Chem. C. 2019, 123, 5782-5789.

(5) Fukuda, M.; Fujii, M.; Hayashi, S. Room-Temperature below Bulk-Si Band Gap Photoluminescence from P and B Co-Doped and Compensated Si Nanocrystals with Narrow Size Distributions. J. Lumin. 2011, 131, 1066-1069.

(6) Kanno, T.; Sugimoto, H.; Fucikova, A.; Valenta, J.; Fujii, M. Single-Dot Spectroscopy of Boron and Phosphorus Codoped Silicon Quantum Dots. J. Appl. Phys. 2016, 120, 164307. Sugimoto, H.; Yamamura, M.; Fujii, R.; Fujii, M. Donor-Acceptor Pair Recombination in Size-Purified Silicon Quantum Dots. Nano Lett. 2018, 18, 7282-7288.

Luo, J.-W.; Stradins, P.; Zunger, A. Matrix-Embedded Silicon Quantum Dots for Photovoltaic Applications: A Theoretical Study of Critical Factors. Energy Environ. Sci. 
2011, 4, 2546.

(9) Dal Negro, L.; Hamel, S.; Zaitseva, N.; Yi, J. H.; Williamson, A.; Stolfi, M.; Michel, J.; Galli, G.; Kimerling, L. C. Synthesis, Characterization, and Modeling of NitrogenPassivated Colloidal and Thin Film Silicon Nanocrystals. IEEE J. Sel. Top. Quantum Electron. 2006, 12, 1151-1163.

(10) Mangolini, L.; Jurbergs, D.; Rogojina, E.; Kortshagen, U. Plasma Synthesis and LiquidPhase Surface Passivation of Brightly Luminescent Si Nanocrystals. J. Lumin. 2006, 121, 327-334.

(11) Limpens, R. Carrier Dynamics in Coupled Silicon Nanocrystal Systems. PhD thesis, University of Amsterdam (UvA), 2016.

(12) Stegner, A. R.; Pereira, R. N.; Klein, K.; Wiggers, H.; Brandt, M. S.; Stutzmann, M. Phosphorus Doping of Si Nanocrystals: Interface Defects and Charge Compensation. Phys. B Condens. Matter 2007, 401-402, 541-545.

(13) Puthen Veettil, B.; Wu, L.; Jia, X.; Lin, Z.; Zhang, T.; Yang, T.; Johnson, C.; McCamey, D.; Conibeer, G.; Perez-Würfl, I. Passivation Effects in B Doped Self-Assembled Si Nanocrystals. Appl. Phys. Lett. 2014, 105, 222108.

(14) Ma, J.; Wei, S.-H.; Neale, N. R.; Nozik, A. J. Effect of Surface Passivation on Dopant Distribution in Si Quantum Dots: The Case of B and P Doping. Appl. Phys. Lett. 2011, 98, 173103.

(15) Klimov, V. I.; Mikhailovsky, A. A.; McBranch, D. W.; Leatherdale, C. A.; Bawendi, M. G. Quantization of Multiparticle Auger Rates in Semiconductor Quantum Dots. Science 2000, 287, 1011-10143. 